\title{
Primary biliary cirrhosis: new perspectives in diagnosis and treatment
}

\author{
Martin I Prince, David E J Jones
}

\begin{abstract}
Primary biliary cirrhosis (PBC) is a chronic autoimmune disease characterised by cholestatic liver function tests, antimitochondrial antibodies, and abnormal liver histology. Early descriptions of a rare rapidly progressive disease no longer reflect the more indolent progress often seen today. Many patients have significant long term morbidity through symptoms such as fatigue and itch with a minority progressing to liver failure and need for transplantation. The current data on the diagnosis, clinical progression, and treatment of PBC are reviewed.

(Postgrad Med f 2000;76:199-206)
\end{abstract}

Keywords: primary biliary cirrhosis; liver transplantation; pruritus

Primary biliary cirrhosis (PBC) is a chronic cholestatic liver disease with a probable autoimmune aetiology. Increasing awareness of PBC among doctors, and the availability and more widespread use of diagnostic tests such as autoantibody screens, has led to an increasing rate of diagnosis. PBC has traditionally been regarded as a severe but rare disorder with a high probability of progression to cirrhosis with the complications of portal hypertension and, ultimately, liver failure. Wider diagnosis has, however, led to an increased recognition of milder forms of the disease where the risk of death from liver related complications is markedly lower. The impact of PBC related symptoms on quality of life, however, remains significant in these patients. Changes in diagnosis, disease severity spectrum and, therefore, goals for treatment have thus forced us to reassess our approach to disease management.

Here we present a review of recent changes

Centre for Liver Research, University of Newcastle, UK M I Prince

D E J Jones

Correspondence to: Dr David E J Jones, Centre for Liver Research, 4th Floor, William Leech Building, Medical School, Framlington Place,

Newcastle upon Tyne NE2 4HH, UK (e-mail: D.E.J.Jones@ncl.ac.uk)

Submitted 10 May 1999 Accepted 27 September 1999 on characteristic findings on liver biopsy obtained at laparotomy performed to exclude other obstructive causes for cholestasis. The discovery of antimitochondrial antibodies (AMA) in the $1960 \mathrm{~s},{ }^{4}$ and their subsequent inclusion in "routine" autoantibody profiles, has led to the diagnosis of PBC being made more frequently and, usually at a much earlier stage in the disease process. Jaundice at the time of diagnosis is now the exception rather than the norm. ${ }^{5-8}$

PBC is classically diagnosed on the basis of the triad of AMA, abnormal liver function tests (LFTs) that are typically cholestatic (with raised alkaline phosphatase levels being the most frequently seen abnormality), and characteristic histological changes ${ }^{9}$ in the absence of extrahepatic biliary obstruction. ${ }^{10}$ Liver biopsy, although desirable, may not be possible in some patients because of infirmity or clotting abnormalities. The very strong association between the presence of AMA and PBC means, however, that in the presence of cholestatic LFTs and AMA, and the absence of other aetiological factors these patients can be regarded as having "probable" PBC. We currently exclude obstruction with transabdominal ultrasound examination and use endoscopic retrograde cholangiopancreatography or magnetic resonance cholangiopancreatography only when the distinction between PBC and extrahepatic obstruction is in doubt (for example where there is a dilated common bile duct or the biopsy suggests coexisting obstruction).

AMA are both the most specific and the most sensitive marker of disease presence and are found in over $95 \%$ of patients with PBC. ${ }^{11-14}$ Nine staining patterns for AMA on immunofluorescence have been described, of which the M2 distribution is the best associated with PBC. Other immunofluorescence staining patterns are also seen (rarely) in other non-autoimmune diseases. ${ }^{15}$ AMA with an M2 distribution have been reported in patients with autoimmune hepatitis ${ }^{16}$ emphasising the importance of obtaining histological confirmation of the diagnosis if possible.

The association between the presence of AMA, particularly in an M2 pattern, and the histological features of PBC appears to be stronger than many of the associations to autoantibodies reported in other autoimmune diseases. Mitchison et al reported a series of 29 patients with serum AMA but normal LFTs who underwent liver biopsy. ${ }^{17}$ Liver histology was diagnostic of PBC in 12 patients, suggestive of PBC in a further 12, and normal in just two. Furthermore, follow up of these patients after 10 years showed that 24 had developed 
persistently abnormal serum LFTs. ${ }^{18}$ The clear implication of these studies is that when AMA is persistently present patients should, even in the absence of clinical features suggestive of the disease or cholestatic LFTs, be considered to have an early ("pre-disease") form of PBC and should undergo regular monitoring to detect the development of overt "classical" PBC.

AMA are directed against highly conserved antigens present on the inner mitochondrial membrane. These antigens have been identified as members of the 2-oxoacid dehydrogenase family of multienzyme complexes (in particular the pyruvate dehydrogenase complex, PDC), all of which play fundamental parts in cell metabolism..$^{19}{ }^{20}$ Intriguingly, the PDC is remarkably conserved throughout evolution, being found in a highly homologous form in all eukaryotic and prokaryotic organisms from rickettsia to man. The pathway leading to breakdown of immune tolerance to such a highly conserved self antigen, the mechanism by which autoreactive $T$ cell responses to PDC and/or AMA result in the characteristic liver damage seen in $\mathrm{PBC}$, and the reason why inflammatory damage in $\mathrm{PBC}$ is largely limited to the liver when every cell in the body contains the PDC remain, however, largely unclear.

A small minority of patients have all the histological, biochemical, and clinical signs of PBC but are persistently AMA negative. The term "autoimmune cholangitis" has recently been coined to describe these patients (who are typically antinuclear antibody or antismooth muscle antibody positive ${ }^{12}$ ), and has largely replaced the term "AMA negative PBC". 11122122 Autoimmune cholangitis appears to run a similar clinical course to "classical" PBC.

The histological changes seen in the liver in PBC are well defined and are traditionally graded into four stages of severity as defined by Scheuer. ${ }^{9}$ These changes are summarised in table 1 . The biliary epithelial cells lining the small intrahepatic bile ducts are the primary target for immunological damage, and thus the majority of early changes are seen in the portal and periportal regions. As only patients in stage IV have a "true" histological cirrhosis, the term primary biliary cirrhosis may be considered a misnomer. The recognition of this, combined with the stigma felt by many patients at being diagnosed with "cirrhosis" has led to recent calls for the term PBC to be replaced. There is, at present, no consensus with regard to suitable alternative terms.

Biochemical testing of patients with PBC typically reveals a cholestatic pattern of LFTs together with raised immunoglobulin $\operatorname{IgM}$ concentrations. ${ }^{67}$ Very early disease may be associated with normal LFTs, ${ }^{17}$ and LFTs may also occasionally normalise over time, particularly when patients are treated with ursodeoxycholic acid (UDCA; see below). ${ }^{23}$ Individuals with PBC and a more hepatitic picture of LFTs are well described. In some cases these patients may have a crossover syndrome between PBC and autoimmune hepatitis. ${ }^{16}$ This possibility should be particularly born in mind when patients also have raised immunoglobulin IgG concentrations and/or antinuclear or smooth muscle antibodies. Such patients may show an excellent response to combination treatment with UDCA and corticosteroids. ${ }^{16}$ The degree of interface hepatitis seen on liver biopsy in such patients is, in our experience, predictive of the response to corticosteroid therapy.

\section{Epidemiology}

Ninety per cent of patients with PBC are female and the majority are diagnosed after the age of $40 .^{7}$ Only one case of paediatric PBC has been reported. Although this girl fulfilled the diagnostic triad for PBC as above, she also had atypical liver immunohistochemical staining patterns and autoantibodies. ${ }^{24}$ There remains debate as to whether this was truly PBC.

The incidence and prevalence of PBC appears to be significantly higher in the family members of PBC patients than in the general population; the sibling relative risk $\left(\lambda_{\mathrm{s}}\right)$ for PBC has recently been calculated as 10.5 in a population based study. ${ }^{25}$ The familial risk of disease development is not, we feel, high enough to warrant screening of relatives. It does, however, provide important clues regarding the aetiology of the disease suggesting there to be a genetic component to disease. ${ }^{26}$

Wide variations in the population prevalence and incidence of PBC have been reported, ${ }^{27}$ although some of the lower estimates may have resulted from incomplete methods of case finding. Two recent studies from the UK have estimated the incidence of PBC at between 204 and 251 per million in the general population and at 954 per million in the group most at risk of disease (women over the age of 40). ${ }^{28}{ }^{29}$ Furthermore, the incidence of PBC appears to be rising. ${ }^{27}$ Such apparent increases may result

Table 1 Histological staging of PBC on liver biopsy (after Scheuer ${ }^{9}$ )

\begin{tabular}{ll}
\hline Stage & Key histological features \\
\hline I: The florid duct lesion & $\begin{array}{c}\text { Expansion of some, but often not all, portal tracts around septal and interlobular bile ducts by aggregates of lymhocytes and } \\
\text { plasma cells. Formation of often poorly defined granulomas within or close to affected tracts } \\
\text { Irregularities or rupture of biliary epithelium, often with relative preservation of remaining portal architecture }\end{array}$ \\
& $\begin{array}{l}\text { Histological evidence of cholestasis usually absent } \\
\text { Changes in all portal tracts. Expansion of portal tracts by immature connective tissue, mononuclear and neutrophil aggregates } \\
\text { and, to a lesser extent than in stage I, by lymphocyte aggregates } \\
\text { II: Ductular proliferation }\end{array}$ \\
& $\begin{array}{l}\text { Bile ducts scanty with proliferation of biliary ductules. Occasional biliary plugging } \\
\text { Inflammation less severe, although the sites of bile ducts may be marked by lymphocyte collections }\end{array}$ \\
III: Septal stage & $\begin{array}{l}\text { Formation of dense fibrous tissue extending from portal tracts, but the absence of regeneration nodules } \\
\text { Cholestasis frequent }\end{array}$ \\
IV: Cirrhosis & Formation of regenrative nodules and a "true" cirrhosis \\
& Gross reduction in number of bile ducts \\
\hline
\end{tabular}


to a significant degree from improved diagnosis and awareness of the disease and the increased use of autoantibody screens in primary care. It is impossible, however, to exclude a genuine rise in the frequency of disease.

Geographical clusters of disease have been reported. The most prominent of these was that found by Triger in Sheffield where $75 \%$ of all cases of PBC obtained their drinking water from a single reservoir which supplied only $20 \%$ of the town's population..$^{31}$ No causative environmental agents were, however, identified in the water supply. Observations such as this, together with the presence of the PDC with significant homology to that seen in humans in all bacteria, has led to the suggestion that exposure to bacterial PDC might, through the process of molecular mimicry, contribute to the breakdown of immune tolerance to self PDC and the induction of AMA. ${ }^{32}$ Given the rarity of AMA and PBC in the population, and the universal exposure to bacterial PDC experienced by the population, additional factors are likely to be required for AMA induction and the development of PBC. One additional factor is likely to be the apparent genetic susceptibility to the disease. Other postulated suggestions have included a putative viral trigger ${ }^{33} 34$ and mycobacteria. ${ }^{35}$ A recently described mouse model of PBC (experimental autoimmune cholangitis) may provide us with further important insights into the immunopathogenesis of the disease. ${ }^{3637}$

\section{Symptoms and associated conditions}

Early reports described PBC as a severe progressive disorder, presenting with itch, with patients often developing the complications of jaundice, liver failure, and premature death. ${ }^{1}$ However, earlier and more widespread diagnosis, possibly together with the introduction of prognosis modifying drugs, has meant that many, if not most, patients present with a milder, more indolent form of disease. Increasingly, case series comprise patients with early stage disease, a significant minority of whom are asymptomatic at diagnosis. ${ }^{3}$ 6-8 3839

As the symptoms of liver failure become rarer other symptoms are predominating, the two most frequently seen being persistent fatigue and itch. Persistent fatigue is the commonest symptom in PBC occurring in up to $80 \%$ of patients. Furthermore, this troubling symptom interferes with normal daily activity in the majority of these patients. ${ }^{8}$ This symptom occurs independently of other problems (for example, anaemia or hypothyroidism $)^{40}{ }^{41}$ and is not related to sleep disturbance. ${ }^{42}$ Fatigue severity does not correlate with other clinical, biochemical, and histological markers of PBC severity. ${ }^{40}{ }^{41}{ }^{43}$ Although fatigue levels correlate weakly with depression inventory scales, the majority of patients with PBC and fatigue are not clinically depressed. ${ }^{41}$ This apparent association may result from items within depression inventories attributing symptoms of chronic liver disease as markers of depression (for example, weight loss, appearance change). The mechanism responsible for fatigue in PBC is poorly understood. ${ }^{44}$ Studies using cholestatic rat models have implicated a central neurochemical process, possibly through hippocampal control of endogenous corticosteroids. ${ }^{45} 46$ Such work has not been repeated in humans.

Itch is the second commonest symptom in PBC occurring in $50 \%-60 \%$ of patients. ${ }^{8}$ Itch is characteristically worse on the palms and soles and is not associated with cutaneous changes (apart from those due to repeated scratching such as lichenification or localised hyperpigmentation), making it distinguishable from other dermatological conditions. Pruritus was classically thought to result from the retention of irritant bile salts which accumulated in the skin. However, itch severity shows only a weak correlation with the degree of cholestasis. Animal models of cholestasis have suggested that pruritus actually results from the accumulation of endogenous opioid agonists and up-regulation of central opioid receptors. ${ }^{47}$ This has led to trials of opiate antagonists for symptomatic therapy (see below)..$^{48}$

PBC is associated with the cutaneous changes of generalised hyperpigmentation and facial xanthelasmata. The latter changes result from raised total cholesterol concentrations. ${ }^{3}$ There does not, however, appear to be an increased incidence of atherosclerotic disease in patients with PBC, presumably as much of the cholesterol rise is due to increases in the high density lipoprotein fraction. ${ }^{50}$

PBC is frequently associated with other autoimmune conditions. The most common of these are Sjögren's syndrome (found in $31 \%$ of patients) and thyroid disease (7\%), ${ }^{51}$ although there are case reports of almost every other autoimmune disease occurring in patients with PBC. The incidence of coeliac disease is increased in PBC. ${ }^{29}$ Steatorrhoea is rare in the absence of coeliac disease. ${ }^{7}$

Osteoporosis is commoner in women with PBC than age and sex matched controls. ${ }^{52}$ The presumed mechanism for this is low grade fat malabsorbtion reducing vitamin $\mathrm{D}$ concentrations and hence inhibiting calcium uptake. Supplementation with parenteral vitamin D does not, however, prevent bone loss. ${ }^{53}$ The true prevalence of osteopenia (bone mineral density more than two standard deviations below age and sex matched norms, without fragility fractures) is unknown. Unpublished data from our own unit suggest that ostoepenia in best assessed by a single screening densitometry measurement at diagnosis followed by a repeat scan either after five years or when liver transplantation is considered. Audit of our patients suggests that this regimen will identify all clinically relevant cases of osteopenia at a much lower cost than annual screening. Coeliac disease should be excluded in all patients with severe osteoporosis. ${ }^{29}$

Concerns have been raised regarding possible associations between PBC and malignant disease, particularly breast and hepatocellular carcinoma. Early reports of an increased risk of breast cancer ${ }^{54} 55$ have not been confirmed in later studies, particularly when the effects of increased surveillance are accounted for. ${ }^{5657}$ 
Hepatocellular carcinoma is markedly increased in PBC (relative risk $=26.5) .{ }^{56}$ However, this risk is very highly concentrated in the relatively small group of male patients with stage IV (that is cirrhotic) disease. ${ }^{58}$ As hepatocellular carcinoma is now the second commonest cause of death in male PBC patients,${ }^{58}$ we advocate routine monitoring with ultrasound and serum $\alpha$-fetoprotein levels in men with known cirrhosis who are young enough to considered for transplantation.

\section{Prognosis}

The prognosis for patients with PBC is heterogeneous. Prognostic models have been studied in some detail with the aim of counselling patients and allowing for optimal timing of liver transplantation. Serum bilirubin was the first variable shown to be related to prognosis. ${ }^{59} \mathrm{It}$ is, however, only raised in a minority of patients and then only in "late stage" disease, with the result that it is of limited usefulness in planning treatment. Several groups have subsequently developed complex prognostic indices producing scores based on clinical variables which can then be used to predict survival over a variety of periods. ${ }^{60-64}$ Three of these models are summarised in table 2. The European model requires recent histology which may not be available in the presence of a coagulopathy and hence the Mayo risk score is perhaps the most widely used of these. The Mayo score has been modified to assess short term (that is less than two year) survival ${ }^{65}$ and has been validated in several centres. ${ }^{66-68}$ Although highly useful in advanced disease, the Mayo score remains relatively insensitive to early disease advancement before the development of severe biochemical abnormalities. Furthermore, the Mayo score is strongly influenced by bilirubin concentrations and was devised before the widespread introduction of treatment with UDCA (which reduces bilirubin concentrations $\left.{ }^{69-73}\right)$. Concerns have been raised as to whether the Mayo score remains valid after UDCA treatment. Two recent studies have addressed this question and reported no significant variation between actual survival and that predicted by the Mayo score in patients treated with UDCA. ${ }^{74} 75$

The prognosis for patients with early and asymptomatic disease is uncertain with most reports being limited to a single centre and therefore subject to Berkson's bias. The prognosis of asymptomatic disease appears to be better than symptomatic. ${ }^{76-78}$ However, asymptomatic patients with PBC still have higher death rates than the age and sex adjusted norm ${ }^{79}$ and, furthermore, between $40 \%$ and $100 \%$ of these patients will subsequently develop symptoms of PBC. ${ }^{18} 7980$ Since increasing numbers of patients are being diagnosed at an asymptomatic stage more information is required on the prognosis in early disease, both to counsel the patient and to weigh the risks and benefits of treatment. AMA subtype, ${ }^{81}$ tumour necrosis factor promoter polymorphisms, ${ }^{82}$ and tests of hepatic metabolic reserve ${ }^{83-87}$ have all been shown to predict the course of disease at an early stage. However, none of these are readily available in the majority of clinical centres.

\section{Treatment}

There are two aims of medical treatment in PBC. The first is to slow progression of the disease to cirrhosis with its attendant complications thereby increasing patient survival (or at least prolonging the time to transplantation). The second is to treat the symptoms of the disease thereby, hopefully, improving quality of life. Liver transplantation in PBC is highly effective in both prolonging life and in eliminating symptoms.

\section{(A) TREATMENTS AIMED AT SLOWING DISEASE} PROGRESSION

Many drugs have used in an attempt to improve prognosis in PBC. These can be broadly divided into two groups of agent, namely exogenous hydrophilic bile acids and immunosupressants. The best studied and most successful of these has been UDCA.

UDCA is a strongly hydrophilic bile acid which is absorbed in the terminal ileum as part of the physiological enterohepatic recirculation. Prolonged treatment results in UDCA replacing a significant proportion $(42 \%)$ of bil-

Table 2 Prognostic indices in PBC

\begin{tabular}{|c|c|c|c|}
\hline Model & Variables included & Formula for calculation of index & Method of application of index \\
\hline European $^{60}$ & $\begin{array}{l}\text { Bilirubin }(\mu \mathrm{mol} / \mathrm{l}) \\
\text { Age (years) } \\
\text { Albumin }(\mathrm{g} / \mathrm{l}) \\
\text { Prothrombin time (sec) } \\
\text { Central cholestasis } \\
\text { Cirrhosis } † \\
\text { Azathioprine }\end{array}$ & $\begin{aligned} \mathrm{R}= & \left(2.51 \times \log _{\mathrm{e}} \text { serum bilirubin }\right)+(0.00069 \times \exp \\
& (\text { age- } 20) / 10)-(0.05 \times \text { albumin })+(0.88 \times \text { cirrhosis })+ \\
& (0.68 \times \text { central cholestasis })+(0.52 \times \text { azathiopirine })+3.09\end{aligned}$ & Cross reference to graph \\
\hline Mayo $^{62}$ & $\begin{array}{l}\text { Bilirubin }(\mathrm{mg} / \mathrm{dl}) \\
\text { Age (years) } \\
\text { Albumin }(\mathrm{g} / \mathrm{dl}) \\
\text { Prorthrombin time (sec) } \\
\text { Oedema } \$\end{array}$ & $\begin{array}{l}\mathrm{R}=\left(0.871 \times \log _{\mathrm{e}} \text { serum bilirubin }\right)-\left(2.53 \times \log _{\mathrm{e}} \text { albumin }\right)+ \\
\quad(0.039 \times \text { age })+\left(0.859 \times \text { oedema }^{\mathrm{e}}{ }^{\mathrm{e}}\right.\end{array}$ & $\begin{array}{l}\text { Probability of survival of time } \mathrm{t}=\mathrm{S}^{\exp (\mathrm{R}-4.07)} \\
\text { where } \mathrm{S}=\quad 0.970 \text { at } 1 \text { year } \\
0.941 \text { at } 2 \text { years } \\
0.774 \text { at } 5 \text { years }\end{array}$ \\
\hline Japanese $^{63}$ & $\begin{array}{l}\text { Age (years) } \\
\text { Bilirubin }(\mathrm{mg} / \mathrm{dl}) \\
\text { Albumin }(\mathrm{g} / \mathrm{dl}) \\
\text { Cholesterol }(\mathrm{mg} / \mathrm{dl})\end{array}$ & $\begin{array}{l}\mathrm{PI}=(0.0409 \times \text { age })+\left(0.7801 \times \log _{\mathrm{e}} \text { serum bilirubin }\right)- \\
\quad\left(0.8016 \times \log _{\mathrm{e}} \text { albumin }\right)+(0.3217 \times \log \text { total serum } \\
\quad \text { cholesterol })\end{array}$ & Cross reference to graph \\
\hline
\end{tabular}

${ }^{\star} 0$ if central cholestasis absent on histology, 1 if present.

to if non-cirrhotic on histology, 1 if cirrhotic.

$\ddagger 0$ if no azathioprine treatment, 1 if treated.

$\$ 0$ if no oedema, 0.5 if oedema repsonsive to diuretics, 1 if non-resposive oedema.

$\mathrm{PI}=$ prognostic index. 
iary bile acids. ${ }^{88}$ The exact mechanism for the action of UDCA is unknown but it is thought to act both by replacing more hepatotoxic hydrophobic bile acids in the bile acid pool and as a choloretic agent stimulating increased biliary flow and reducing stagnation. ${ }^{89}{ }^{90}$ In addition, UDCA has been reported to modify biliary epithelial HLA expression ${ }^{91}$ and may therefore have a local immunosuppressant action.

There have been four large double blind randomised controlled trials of UDCA treatment in PBC. ${ }^{69} 717292$ These trials used UDCA at doses of $10-15 \mathrm{mg} / \mathrm{kg}$ and monitored patients over two to four years. The results of three of these have been subjected to a combined analysis. ${ }^{89}$ Although LFTs (particularly bilirubin) improved in all trials, the overall effect on liver transplant free survival was small and no significant differences were found. A small improvement in survival was reported in the combined analysis ${ }^{89}$ with 25 of 273 patients dying in the treatment arm compared with 34 out of 275 in the control arm (with 22 and 32 needing liver transplants respectively). However, this combined analysis did not use preset criteria for selecting studies and may therefore be subject to considerable selection bias. Bateson and Gedling recently described 10 years of clinical experience of prescribing UDCA and reported an improved survival compared to historical controls. ${ }^{93}$

There has been debate as to whether the biochemical improvement predicts a true improvement in survival (see above). This controversy has recently been highlighted by the publication of the preliminary results of a thorough meta-analysis which failed to find any survival benefit for UDCA. ${ }^{94}$

UDCA has a small beneficial effect on pruritus (with $39 \%$ of patients reporting a improvement), but little effect on fatigue. ${ }^{95}$ A small number of patients suffer a paradoxical increase in pruritus with UDCA (up to $10 \%$ of patients in one study ${ }^{95}$ ) which may necessitate stopping treatment. UDCA does not modify the course of associated autoimmune diseases. $^{51}$

In view of the above data it remains controversial as to whether UDCA should be prescribed to all or just a subgroup of patients. Our current practice is to use UDCA in all patients with troubling pruritus and those with a higher probability of developing complications of disease (that is patients who are younger at diagnosis, male, have a raised bilirubin or have a liver biopsy specimen showing active inflammation or development of fibrosis).

Immunosupressant treatments used in PBC have included penicillamine, ${ }^{96}{ }^{97}$ azathioprine, ${ }^{60}$ methotrexate, ${ }^{98} 99$ cyclosporin, ${ }^{100}$ and colchicine. ${ }^{101-103}$ Although methotrexate is still used in some American centres, none of these drugs remain in regular clinical usage in the UK because limited effectiveness and/or the risk of side effects. Prednisolone has been shown to be effective in improving the biochemical markers of PBC, ${ }^{104}$ its use has, however, been limited by concerns regarding its detrimental effects on osteoporosis. We currently use steroids only in patients whom we feel may have several features to suggest a hepatitis crossover picture (that is unusually high transaminase level, high IgG levels, high titre of antinuclear antibodies, and/or severe interface hepatitis on liver biopsy) or those with a poor medium term prognosis in whom transplantation is not an option or could usefully be delayed (for example for personal reasons). Our standard regimen is to start patients on 30 $\mathrm{mg}$ of prednisolone daily for two to four weeks to assess respones and then tail the dose down to between 5 and $10 \mathrm{mg}$ daily maintainance provided side effects remain acceptable.

\section{(B) TREATMENTS AIMED AT REDUCING THE} SYMPTOMS OF PBC

Pruritus may be treated with a number of agents. Of these the bile acid sequestrants are the most widely used. Most units now use cholestyramine as a first line agent, although the development of (usually gastrointestinal) side effects often limits treatment. These side effects can be minimised by starting at very low dosages (for example, half a sachet once daily) and gradually titrating upwards to a level where itch is controlled at an acceptable level of palatability. Colestipol may be tried in patients who cannot tolerate cholestyramine. UDCA improves itch in up to $40 \%$ of patient. ${ }^{95}$

A number of third line options exist for patients for whom the above treatments are either unsuitable or ineffective. Rifampicin relieves itch in up to $89 \%$ of patients. ${ }^{105} 106$ The actions of rifampicin do not appear to related to its liver enzyme inducing capacity as other enzyme inducers (with the possible exception of phenobarbitone) are ineffective in controlling pruritus in PBC. Rifampicin can, however, induce hepatitis in its own right and its use must therefore be closely monitored with serial liver function testing. Our current practice is to start rifampicin at a dose of $150 \mathrm{mg}$ daily and increase the dose in increments of $150 \mathrm{mg}$ to a maximum of $450 \mathrm{mg}$ daily or until itch is relieved. We monitor LFTs monthly, although drug induced hepatitis has been rare in our experience. We have safely continued the drug long term where symptoms have necessitated this.

Phenobarbitone is less effective than rifampicin at relieving itching and its neurological side effects usually preclude its use for this indication. ${ }^{106}$ Antihistamines are not recommended for itch because of concerns regarding accumulation in cholestasis and fatigue induction. A case series of five patients reported itch relief with grapefruit juice (at a dose of 400 $\mathrm{ml} /$ day), ${ }^{107}$ although this has never been subject to a controlled trial. Early reports of ondansetron being effective for this symptom have not been supported by clinical trials. ${ }^{108}$

Opiate antagonists may be tried as a last line to prevent itch before consideration for liver transplantation. ${ }^{48}$ Our current protocol for using opiate antagonists is to offer an in patient overnight trial of intravenous naloxone at a dose of $0.2 \mu \mathrm{g} / \mathrm{kg} / \mathrm{min}$ (both to assess responsiveness and side effects) and, if appropriate, to 


\section{Questions (correct answers at end of article)}

1. Which of the following are thought to be responsible for the itch associated with PBC?

a. Raised serum bilirubin

b. Bile salts retained in the skin

c. Endogenous opioid agonists

d. Raised cholesterol

e. Autoantibodies

2. The AMA seen in PBC react against which autoantigen:

a. The outer mitochondrial membrane

b. The pyruvate dehydrogenase complex

c. Mitochondrial DNA

d. Bilirubin

e. Mitochondrial cytochrome systems

3. The commonest symptom in PBC is:

a. Jaundice

b. Fatigue

c. Itch

d. Right upper quadrant pain

e. Bone pain

4. Which of the following medications have not been used in trials of prognostic benefit in $\mathrm{PBC}$ ?

a. Cyclosporin A

b. Ursodeoxycholic acid

c. Colchicine

d. Methotrexate

e. Rifampicin

5. Which of following are not seen at an increased frequency in patients with $\mathrm{PBC}$ ?

a. Ischaemic heart disease

b. Hepatocellular carcinoma

c. Osteoporosis

d. Rheumatoid arthritis

e. Hypercholesterolemia

follow this with the long acting oral antagonist naltrexone. This is a non-licensed indication and should be used with caution as there are no long term data regarding its use in PBC.

Although fatigue is the most troubling symptom for the majority of patients, ${ }^{8}$ there is little one can do to treat it. There have been no reported randomised placebo controlled trials specifically aimed at improving this symptom. This may partly be due to perceived difficulties in quantifying this symptom, although validated measures of its severity do exist. ${ }^{40}$ One should always exclude associated conditions such as anaemia, hypothyroidism, or renal impairment. Antidepressants may be tried in view of the association between fatigue and subclinical depression, although this treatment is not supported by trial evidence. Even in patients who attribute their fatigue to poor sleep, hypnotics have no place in the treatment of fatigue. ${ }^{42}$ Anecdotal reports have suggested benefit from oral antioxidant medication. ${ }^{109}$

Osteoporosis should be treated as in patients without PBC. We no longer routinely treat all patients with intramuscular vitamin $\mathrm{D}$ as its effect is limited in patients without clinical malabsorbtion. ${ }^{53}$ Established osteoporosis should be treated with bisphosphonates. Many doctors have concerns about treating postmenopausal women with oestrogen containing hormone replacement therapy in view of possible cholestatic side effects. A recent case series, however, showed these drugs to be safe and effective at improving bone mineral density. ${ }^{110}$ It is still probably prudent to monitor LFTs more closely for the first few months of hormonal treatment.

Although in the past it was conventional to give all patients with PBC paraenteral fat soluble vitamins (notably $\mathrm{A}, \mathrm{D}$, and $\mathrm{K}$ ), this is no longer our practice as vitamin deficiency is rare in the absence of frank fat malabsorbtion. As stated above there is little evidence to support regular prophylactic use of vitamin D and calcium supplements to prevent osteoporosis.

(C) LIVER TRANSPLANTATION

Transplantation remains the only effective treatment for advanced disease with complications of liver failure and may also be needed for intractable symptoms. ${ }^{11}$ Many patients are precluded from transplantation by age or infirmity at the time of deterioration. PBC currently accounts for approximately $20 \%$ of liver transplants performed in the UK (1998 UK Transplant Special Support Authority data, personal communication) and has the best outcome of any indication for this operation. Current survival rates are approximately $80 \%-90 \%$ at one year and $70 \%$ at five years. Survival after transplantation is significantly worse for patients who have jaundice, renal impairment, or ascites ${ }^{112}$ before surgery. There is therefore a careful balancing act to be undertaken in all patients with advancing disease; delaying operation as long as possible to prevent unnecessary transplants, but operating before transplant associated mortality is significantly increased by any of the above factors. In practice this is best done by regular calculation of prognostic indices such the Mayo score. Patients may be considered for transplantation when their predicted survival falls below that expected after operation. In practice many gastroenterolgists refer any patient under the age of 65 years for monitoring at a transplant centre when their bilirubin exceeds $50 \mu \mathrm{mol} / 1$ without a reversible cause.

Transplantation does not cure patients. Recurrence of AMA is almost universal and some patients will develop histological changes of recurrent PBC. ${ }^{111}$ It is unknown whether recurrence can be prevented by differing immunosupression regimens or by deliberate HLA mismatching between donor and recipient. Quality of life is, however, markedly improved by transplantation with a high degree 
of patient satisfaction. ${ }^{113}$ The efficacy of transplantation in prolonging life improving quality of life means that all patients with advanced disease who do not have physical contraindications should be at least referred for transplant assessment.

\section{Summary}

PBC has moved from being a rare but rapidly fatal disease to being an uncommon chronic debilitating, but not necessarily fatal, condition. Increased diagnostic awareness and/or increases in true incidence have led to an increased prevalence of PBC. The chronic morbidity, treatment costs, and transplantation needs of patients with PBC mean that this disease now accounts for a sizeable proportion of clinical hepatology workload. As prognosis is improved by earlier diagnosis and therapy more emphasis needs to be placed on symptomatic treatment.

1 Ahrens E, Payne M, Kunkel H, et al. Primary biliary cirrhosis. Medicine 1950;29:299-366.

2 Addison T, Gull W. On a certain affliction of the skin-vitiligoides-a planus tuberosa. Guys Hospital Review skin-vitiligoid

3 Sherlock S, Scheuer P. The presentation and diagnosis of 100 patients with primary biliary cirrhosis. $N$ Engl f Med 1973;289:674-8.

4 Walker J, Doniach D, Roitt I, et al. Serological tests in diagnosis of primary biliary cirrhosis. Lancet $1965 ; \mathbf{i}: 827-33$

5 Eriksson S, Lindgren S. The prevalence and clinical spectrum of primary biliary cirrhosis in a defined population. Scand F Gastroenterol 1984;19:971-6.

6 Christensen E, Crowe J, Doniach D, et al. Clinical pattern and course of disease in primary biliary cirrhosis based on an analysis of 236 patients. Gastroenterology 1980;78:23646 .

7 James O, Macklon AF, Watson AJ. Primary biliary cirrhosis-a revised clinical spectrum. Lancet 1981;i:127881 .

8 Witt-Sullivan $\mathrm{H}$, Heathcote J, Cauch $\mathrm{K}$, et al. The demography of primary biliary cirrhosis in Ontario, Canada. Hepatology 1990;12:98-105.

9 Scheuer P. Primary biliary cirrhosis. Proceedings of the Royal Society of Medicine 1967;40:1257.

10 Kaplan M. Primary biliary cirrhosis. $N$ Engl $f \mathrm{Med}$ 1987;316:521-8.

11 Invernizzi P, Crosignani A, Battezzati PM, et al. Comparison of the clinical features and clinical course of antimitochondrial antibody-positive and negative primary biliary cirrhosis. Hepatology 1997;25:1090-5.

12 Lacerda MA, Ludwig J, Dickson ER, et al. Antimitochondrial antibody-negative primary biliary cirrhosis. Am f Gastroenterol 1995;90:247-9.

13 Mattalia A, Quaranta S, Leung PS, et al. Characterization of antimitochondrial antibodies in health adults. Hepatology 1998;27:656-61.

14 Turchany JM, Uibo R, Kivik T, et al. A study of antimitochondrial antibodies in a random population in Estonia. Am f Gastroenterol 1997;92:124-6.

15 Berg PA, Doniach D, Roitt IM. Mitochondrial antibodies in primary biliary cirrhosis. I. Localization of the antigen to mitochondrial membranes. F Exp Med 1967;126:277-90.

16 Chazouilleres O, Wendum D, Serfaty L, et al. Primary biliary cirrhosis-autoimmune hepatitis overlap syndrome: clinical features and response to therapy. Hepatology 1998;28:296-301.

17 Mitchison HC, Bassendine MF, Hendrick A, et al. Positive antimitochondrial antibody but normal alkaline phosphatase: is this primary biliary cirrhosis? Hepatology phosphatase: is

18 Metcalf J, Mitchison H, Palmer J, et al. Natural history of early primary biliary cirrhosis. Lancet 1996;348:1399-402 .

19 Gershwin M, Mackay I, Sturgess A, et al. Identification of specificity of a cDNA encoding the $70 \mathrm{kD}$ mitochondrial antigen recognised in primary biliary cirrhosis. F Immuno 1987; 138:3525-31

20 Yeaman S, Fussey S, Danner D, et al. Primary biliary cirrhosis: identification of two common M2 mitochondrial autoanitgens. Lancet 1988;i:1067-70.

21 Michieletti P, Wanless IR, Katz A, et al. Antimitochondria antibody negative primary biliary cirrhosis: a distinct syndrome of autoimmune cholangitis. Gut 1994;35:260-5.

22 Goodman ZD, McNally PR, Davis DR, et al. Autoimmune cholangitis: a variant of primary biliary cirrhosis. Clinicopathologic and serologic correlations in 200 cases. Dig Dis Sci 1995; 40:1232-42.

23 Jorgensen RA, Dickson ER, Hofmann AF, et al. Characterisation of patients with a complete biochemical response to ursodeoxycholic acid. Gut 1995;36:935-8.
24 Melegh B, Skuta G, Pajor L, et al. Autoantibodies against subunits of pyruvate dehydrogenase and citrate synthase in a case of paediatric biliary cirrhosis. Gut 1998;42:753-6.

25 Jones D, Watt F, Metcalf J, et al. Familial primary biliary cirrhosis: a geographically based population study. $\mathcal{F}$ Hepatol 1999;30:402-7.

26 Agarwal K, Jones D, Bassendine M. Genetic susceptability to primary biliary cirrhosis. Eur f Gastroenterol Hepatol 1999 (in press).

27 Metcalf J, James O. The geoepidemiology of primary biliary cirrhosis. Semin Liv Dis 1996;17:13-22.

28 Metcalf J, Bhopal R, Gray J, et al. Incidence and prevalence of primary biliary cirrhosis in the city of Newcastle upon Tyne, England. Int f Epidemiol 1997;26:830-6.

29 Kingham JG, Parker DR. The association between primary biliary cirrhosis and coeliac disease: a study of relative prevalences. Gut 1998;42:120-2

30 James OF, Bhopal R, Howel D, et al. Primary biliary cirrhosis once rare, now common in the United Kingdom? Hepatology 1999;30:390-4.

31 Triger D. Primary biliary cirrhosis: an epidemiological study. $B M \mathcal{F} 1980 ; 281: 772-5$.

32 Butler P, Valle F, Hamilton-Miller JM, et al. M2 mitochondrial antibodies and urinary rough mutant bacteria in patients with primary biliary cirrhosis and in patients with patients with primary biliary cirrhosis and in patien

33 Meilof JF, Smeenk RJ. Detection of retroviral antibodies in primary biliary cirrhosis [letter]. Lancet 1998;352:739-40.

34 Mason AL, Xu L, Guo L, et al. Detection of retroviral antibodies in primary biliary cirrhosis and other idiopathic biliary disorders. Lancet 1998;351:1620-4.

35 Vilagut L, Vila J, Vinas O, et al. Cross-reactivity of anti-Mycobacterium gordonae antibodies with the major mitochondrial autoantigens in primary biliary cirrhosis [see comments]. F Hepatol 1994;21:673-7.

36 Jones DE, Palmer JM, Yeaman SJ, et al. Breakdown of tolerance to pyruvate dehydrogenase complex in experimental autoimmune cholangitis: a mouse model of primary biliary cirrhosis. Hepatology 1999;30:65-70.

37 Bassendine M, Palmer J, Decruz D, et al. Approaches to a murine model of AMA positive non-suppurative destructive cholangitis (NSDC). F Hepatol 1998;28:124.

38 Heathcote E. The clinical expression of primary biliary cirrhosis. Semin Liv Dis 1996;17:23-33.

39 Long RG, Scheuer PJ, Sherlock S. Presentation and course of asymptomatic primary biliary cirrhosis. Gastroenterology 1977;72:1204-7.

40 Prince M, James O, Jones D. Fatigue in patients with primary biliary cirrhosis: validation of fatigue severity score as a clinical and research tool. Hepatology 1998;28:542A

41 Cauch-Dudek K, Abbey S, Stewart DE, et al. Fatigue in primary biliary cirrhosis. Gut 1998;43:705-710.

42 Cauch-Dudek K, Heathcote E, Hutere N, et al. Is disturbed sleep, based on objective polysomnographic indicators, a factor in the fatigue of patients with primary biliary cirrhosis. Hepatology 1998;28:A543.

43 Wanless I, Heathcote E, Cauch K. Detailed histological grading as a predictor of morbidity and mortality in primary biliary cirrhosis. Hepatology 1993;18:174A.

44 Jones E. Fatigue associated with chronic liver disease: wrapped inside a mystery inside an enigma. Hepatology $1995 ; 22: 1606-8$

45 Swain MG, Le T. Chronic cholestasis in rats induces anhedonia and a loss of social interest. Hepatology 1998;28:6-10.

46 Swain M, Maric M. Improvement in cholestasis-associated fatigue with a serotonin receptor agonist using a novel rat model of fatigue assessment. Hepatology 1997;25:291-4.

47 Bergasa N, Jones E. The pruritus of cholestasis: potential Bergasa N, Jones E. The pruritus of cholestasis: potential
pathogenic and therapeutic implications of opioids. Gastroenterology 1995; 108:1582-8.

48 Bergasa NV, Talbot TL, Alling DW, et al. A controlled trial of naloxone infusions for the pruritus of chronic cholestasis. Gastroenterology 1992;102:544-9.

49 Wolfhagen FH, Sternieri E, Hop WC, et al. Oral naltrexone treatment for cholestatic pruritus: a double-blind, placebocontrolled study. Gastroenterology 1997;113:1264-9.

50 Crippin JS, Lindor KD, Jorgensen R, et al. Hypercholesterolemia and atherosclerosis in primary biliary cirrhosis: what is the risk? Hepatology 1992;15:858-62.

51 Zukowski TH, Jorgensen RA, Dickson ER, et al. Autoimmune conditions associated with primary biliary cirrhosis: response to ursodeoxycholic acid therapy. Am $\mathcal{F}$ Gastroenterol 1998;93:958-61.

52 Eastell R, Dickson ER, Hodgson SF, et al. Rates of vertebral bone loss before and after liver transplantation in women with primary biliary cirrhosis. Hepatology 1991;14:296-300.

53 Matloff DS, Kaplan MM, Neer RM, et al. Osteoporosis in primary biliary cirrhosis: effects of 25-hydroxyvitamin D3 treatment. Gastroenterology 1982;83(1 pt 1):97-102.

54 Wolke AM, Schaffner F, Kapelman B, et al. Malignancy in primary biliary cirrhosis. High incidence of breast cancer in affected women. Am $\mathcal{F}$ Med 1984;76:1075-8.

55 Goudie BM, Burt AD, Boyle P, et al. Breast cancer in women with primary biliary cirrhosis. BMF 1985;291:1597-

56 Howel D, Metcalf J, James O. Is cancer commoner in primary biliary cirrhosis? Hepatology 1998;28:540A

57 Loof L, Adami HO, Sparen P, et al. Cancer risk in primary biliary cirrhosis: a population-based study from Sweden. Hepatology 1994;20(1 pt 1):101-4.

58 Jones DE, Metcalf JV, Collier JD, et al. Hepatocellular carcinoma in primary biliary cirrhosis and its impact on outcomes. Hepatology 1997;26:1138-42. 
59 Shapiro JM, Smith H, Schaffner F. Serum bilirubin: a prognostic factor in primary biliary cirrhosis. Gut 1979:20:13740 .

60 Christensen E, Neuberger J, Crowe J, et al. Beneficial effect of azathioprine and prediction of prognosis in primary biliary cirrhosis. Final results of an international trial. Gastroenterology 1985;89:1084-91.

61 Christensen E, Altman DG, Neuberger J, et al. Updating prognosis in primary biliary cirrhosis using a timedependent Cox regression model. PBC1 and PBC2 trial groups. Gastroenterology 1993;105:1865-76.

62 Dickson ER, Grambsch PM, Fleming TR, et al. Prognosis in primary biliary cirrhosis: model for decision making. Hepatology 1989;10:1-7.

63 Inoue K, Hirohara J, Nakano T, et al. Prediction of prognosis of primary biliary cirrhosis in Japan. Liver 1995;15:70-7.

64 Wiesner RH, Porayko MK, Dickson ER, et al. Selection and timing of liver transplantation in primary biliary cirrhosis
and primary sclerosing cholangitis. Hepatology 1992;16: and primar $1290-9$.

65 Murtaugh PA, Dickson ER, Van Dam GM, et al. Primary biliary cirrhosis: prediction of short-term survival based on repeated patient visits. Hepatology 1994;20(1 pt 1):126-34

66 Bonsel GJ, Klompmaker IJ, van't Veer F, et al. Use of prognostic models for assessment of value of liver transpla
in primary biliary cirrhosis. Lancet 1990;335:493-7.

in primary biliary cirrhosis. Lancet 1990;335:493-7.
67 Grambsch PM, Dickson ER, Kaplan M, et al. Extramural cross-validation of the Mayo primary biliary cirrhosis survival model establishes its generalizability. Hepatology 1989;10:846-50.

68 van Dam GM, Verbaan BW, Therneau TM, et al. Primary biliary cirrhosis: Dutch application of the Mayo model before and after orthotopic liver transplantation. Hepatogas-

69 Combes B, Carithers RL Jr, Maddrey WC, et al. A randomized, double-blind, placebo-controlled trial of ursodeoxycholic acid in primary biliary cirrhosis. Hepatology 1995;22:759-66.

70 Eriksson LS, Olsson R, Glauman H, et al. Ursodeoxycholic acid treatment in patients with primary biliary cirrhosis. A acid treatment in patients with primary biliary cirrhosis. A study. Scand $\mathcal{F}$ Gastroenterol 1997;32:179-86.

71 Lindor KD, Therneau TM, Jorgensen RA, et al. Effects of ursodeoxycholic acid on survival in patients with primary biliary cirrhosis. Gastroenterology 1996;110:1515-8.

72 Poupon RE, Balkau B, Eschwege E, et al. A multicenter, controlled trial of ursodiol for the treatment of primary biliary cirrhosis. UDCA-PBC Study Group. $N$ Engl $\mathcal{F}$ Med 1991;324:1548-54

73 Turner IB, Myszor M, Mitchison HC, et al. A two year controlled trial examining the effectiveness of ursodeoxycholic acid in primary biliary cirrhosis. 7 Gastroenterol Hepatol 1994;9:162-8

74 Kilmurry MR, Heathcote EJ, Cauch-Dudek K, et al. Is the Mayo model for predicting survival useful after the introduction of ursodeoxycholic acid treatment for primary introduction of ursodeoxycholic acid treatment
biliary cirrhosis? Hepatology 1996;23:1148-53.

75 Bonnard A, Hepatology $1996 ; 23: 1148-53$. significance of bilirubin levels under ursodeoxycholic acid therapy in patients with primary biliary cirrhosis. Hepatology 9.39-43.

76 Beswick DR, Klatskin G, Boyer JL. Asymptomatic primary biliary cirrhosis. A progress report on long-term follow-up and natural history. Gastroenterology 1985;89:267-71.

77 Mahl TC, Shockcor W, Boyer JL. Primary biliary cirrhosis: survival of a large cohort of symptomatic and asymptomatic

patients followed for 24 years. F Hepatol 1994;20:707-13.

78 Roll J, Boyer JL, Barry D, et al. The prognostic importance of clinical and histologic features in asymptomatic and symptomatic primary biliary cirrhosis. $N$ Engl $f \mathrm{Med}$ 1983;308:1-7.

79 Balasubramaniam K, Grambsch PM, Wiesner RH, et al. Diminished survival in asymptomatic primary biliary cirrhosis. A prospective study. Gastroenterology 1990;98: cirrhosis.

80 Nyberg A, Loof L. Primary biliary cirrhosis: clinical features and outcome, with special reference to asymptomatic and outcome, with special reference to
disease. Scand $\mathcal{F}$ Gastroenterol 1989;24:57-64

81 Klein R, Pointner H, Zilly W, et al. Antimitochondrial antibody profiles in primary biliary cirrhosis distinguish at early stages between a benign and a progressive course: prospective study on 200 patients followed for 10 years. Liver 1997;17:119-28

82 Jones D, Watt F, Grove J, et al. Tumour necrosis factor-alpha promoter polymorphism in primary biliary cirrhosis. $f$ Hepatol 1999;30:232-36.

83 Nyberg A, Engstrom-Laurent A, Loof L. Serum hyaluronate in primary biliary cirrhosis - a biochemical marker for progressive liver damage. Hepatology 1988;8:142-6.

84 Eriksson S, Zettervall O. The N-terminal propeptide of collagen type III in serum as a prognostic indicator in primary biliary cirrhosis. $\mathcal{F}$ Hepatol 1986;2:370-8.

85 Reichen J, Widmer T, Cotting J. Accurate prediction of death by serial determination of galactose elimination capacity in primary biliary cirrhosis: a comparison with the capacity in primary biliary cirrhosis: a compa
Mayo model. Hepatology 1991;14:504-10.
86 Beukers R, van Zanten RA, Schalm SW. Serial determination of type III procollagen amino propeptide serum levels in patients with histologically progressive and nonprogressive primary biliary cirrhosis. F Hepatol 1992;14:22-

87 Clements D, Elias E, McMaster P. Preliminary study of indocyanine green clearance in primary biliary cirrhosis. Scand f Gastroenterol 1991;26:119-23.

88 Lindor KD, Lacerda MA, Jorgensen RA, et al. Relationship between biliary and serum bile acids and response to ursodeoxycholic acid in patients with primary biliary cirrhosis. Am f Gastroenterol 1998;93:1498-504.

89 Poupon RE, Lindor KD, Cauch-Dudek K, et al. Combined analysis of randomized controlled trials of ursodeoxycholic acid in primary biliary cirrhosis. Gastroenterology 1997;113: 884-90.

90 Poupon RE, Chretien Y, Poupon R, et al. Serum bile acids in primary biliary cirrhosis: effect of ursodeoxycholic acid therapy. Hepatology 1993;17:599-604.

91 Terasaki S, Nakanuma Y, Ogino H, et al. Hepatocellular and biliary expression of HLA antigens in primary biliary cirrhosis before and after ursodeoxycholic acid therapy. $\mathrm{Am}$ f Gastroenterol 1991;86:1194-9.

92 Heathcote EJ, Cauch-Dudek K, Walker V, et al. The Canadian multicenter double-blind randomized controlled trial of ursodeoxycholic acid in primary biliary cirrhosis. Hepatology 1994;19:1149-56.

93 Bateson MC, Gedling P. Ursodeoxycholic acid therapy for primary biliary cirrhosis. A 10-year British single-centre population-based audit of efficacy and survival. Postgrad Med $f 1998 ; 74: 482-5$.

94 Goulis J, Lerandro G, Burroughs A. No evidence for ursodeoxycholic acid therapy in primary biliary cirrhosis: a meta-analysis. Fournal of Hepatology 1999;30 (suppl 1):57.

95 Lindor KD, Dickson ER, Baldus WP, et al. Ursodeoxycholic acid in the treatment of primary biliary cirrhosis. Gastroenterology 1994;106:1284-90.

96 Bodenheimer HC Jr, Schaffner F, Sternlieb I, et al. A prospective clinical trial of D-penicillamine in the treatment of primary biliary cirrhosis. Hepatology 1985;5:1139-42.

97 Neuberger J, Christensen E, Portmann B, et al. Double blind controlled trial of d-penicillamine in patients with primary biliary cirrhosis. Gut 1985;26:114-9.

98 Kaplan MM, Knox TA. Treatment of primary biliary cirrhosis with low-dose weekly methotrexate. Gastroenterology $1991 ; 101: 1332-8$

99 Kaplan MM, DeLellis RA, Wolfe HJ. Sustained biochemical and histologic remission of primary biliary cirrhosis in response to medical treatment [see comments]. Ann Intern Med 1997;126:682-8.

100 Lombard M, Portmann B, Neuberger J, et al. Cyclosporin A treatment in primary biliary cirrhosis: results of a long-term placebo controlled trial. Gastroenterology 1993; 104:519-26

101 Poupon RE, Huet PM, Poupon R, et al. A randomized trial comparing colchicine and ursodeoxycholic acid combination to ursodeoxycholic acid in primary biliary cirrhosis. UDCA-PBC Study Group. Hepatology 1996;24:1098-103.

102 Kaplan MM, Alling DW, Zimmerman HJ, et al. A prospective trial of colchicine for primary biliary cirrhosis. $N$ Engl f Med 1986;315:1448-54.

103 Bodenheimer H Jr, Schaffner F, Pezzullo J. Evaluation of colchicine therapy in primary biliary cirrhosis. Gastroenterology 1988;95:124-9.

104 Mitchison HC, Palmer JM, Bassendine MF, et al. A controlled trial of prednisolone treatment in primary biliary cirrhosis. Three-year results. F Hepatol 1992;15:336-44.

105 Ghent CN, Carruthers SG. Treatment of pruritus in primary biliary cirrhosis with rifampin. Results of a doubleblind, crossover, randomized trial. Gastroenterology 1988;94: 488-93.

106 Bachs L, Pares A, Elena M, et al. Comparison of rifampicin with phenobarbitone for treatment of pruritus in biliary cirrhosis. Lancet 1989;i:574-6.

107 Horsmans Y, Geubel A. Pruritus associated with chronic liver disease. Ann Intern Med 1996;125:701.

108 O'Donohoue J, Haigh C, Williams R. Ondansetron in the treatment of the pruritus of cholestasis: a randomised controlled trial. Gut 1997;40(S1):A12.

109 Watson J, Jones D, James O, et al. Oral antioxidant therapy for the treatment of primary biliary cirrhosis: a pilot study. $\mathcal{F}$ Gastroenterol Hepatol 1999 (in press).

110 Castedal M, Olsson R, Mattson L-A, et al. Estrogengestagen therapy for low bone mineral density in primar biliary cirrhosis. Hepatology 1998;28(4 suppl):544A.

111 Neuberger J. Transplantation for primary biliary cirrhosis. Semin Liv Dis 1997;17:137-46.

112 Neuberger J, Altman DG, Polson R, et al. Prognosis after liver transplantation for primary biliary cirrhosis. Transplantation 1989;48:444-7.

113 Gross CR, Malinchoc M, Kim WR, et al. Quality of life before and after liver transplantation for cholestatic liver disease. Hepatology 1999;29:356-64.

Answers: 1c, 2b, 3b, 4e, 5a. 